\title{
Diffusion treatment of quantum mechanics and its consequences
}

\author{
Zahid Zakir (1) \\ Centre for Theoretical Physics and Astrophysics, Tashkent, Uzbekistan \\ zzakir@qgph.org
}

\begin{abstract}
Localized ensemble of free microparticles spreads out as in a frictionless diffusion satisfying the principle of relativity. An ensemble of classical particles in a fluctuating classical scalar field diffuses in a similar way, and this analogy is used to formulate diffusion quantum mechanics (DQM). DQM reproduces quantum mechanics for homogeneous and gravity for inhomogeneous scalar field. Diffusion flux and probability density are related by Fick's law, diffusion coefficient is constant and invariant. Hamiltonian includes a "thermal" energy, kinetic energies of drift and diffusion flux. The probability density and the action function of drift form a canonical pair and canonical equations for them lead to the Hamilton-Jacobi-Madelung and continuity equations. At canonical transformation to a complex probability amplitude they form a linear Schrödinger equation. DQM explains appearance of quantum statistics, rest energy ("thermal" energy) and gravity ("thermal" diffusion) and leads to a low mass mechanism for composite particles.
\end{abstract}

\section{INTRODUCTION}

Quantum mechanics is based on mathematical axioms, and not on physical principles following from experiments, and therefore it is, until now, represented only a successful mathematical model. The derivation of its formalism from physical principles was one of the main problems of quantum physics, since this would complete the long time history of the formation of quantum mechanics as a fundamental physical theory. The key step towards this was to find a physical mechanism leading to the quantum mechanical formalism.

In fact, the first step in this direction was the Madelung's hydrodynamic form of the Schrödinger equation [1], and the second step - the discovery by Ehrenfest [2], Schrödinger [3] and Furth [4] of an analogy with some diffusion process.

They showed that free microparticles not only fluctuate, but also the probability density in their initially localized ensemble spreads out in space as some diffusion process. Such diffusion should be frictionless, i.e. conservative, as required by the principle of relativity (i.e. should occur similarly in any inertial frame), and by the time reversal symmetry. Really, the group velocity of a wave packet, describing this process in quantum mechanics, remains constant.

One of the first attempts to find a conservative diffusion in classical systems was the stochastic treatment [5,6,7], based on a combination of two families of Brownian motions. However, it turned out that quantum mechanics cannot be reduced to such simple Markov processes [8-9]. Nevertheless, this attempt led to the formalism of conservative diffusion [7] weakly depending on the mechanism of fluctuations.

The analogy with diffusion in its integral representation also underlies the Feynman's path integral [10], a complex version of the Wiener integral for ordinary diffusion. But this mathematical method also had no physical explanation.

All these attempts were based on a common idea about a fluctuating classical background field, at interaction with which the classical particles acquire quantum properties.
Classical character of the particles and the background field radically simplified the situation, since this excluded that element of irrationalism of the standard interpretation, when "quantum" particles supposedly had the ability to fluctuate in an empty and homogeneous Euclidean space without an external cause, spontaneously changing their energy and momentum. Naive attempts to ascribe the appearance of probabilities to a certain observer brought this irrationalism to the point of absurdity, since this observer was credited with the supreme ability, for example, to create atomic levels for all atoms in the entire universe and, moreover, at all times. Fluctuations of a classical field, as an extended object, are more natural and acceptable from the physical point of view, since a spontaneous increase in the field's energy in one place can be compensated for by the same decrease in other places.

In any treatment that deduces quantum mechanics from some more general physical principles, quantum mechanics should not change at describing known experiments, as Bohr believed, but should be supplemented by some physical mechanism, from which its formalism follows, as Einstein assumed [11]. Fluctuations of microparticles and the diffusion behavior of their ensemble, similar in any inertial frame, indicate that this mechanism can be a fluctuating classical field as a source of fluctuations of particles, and that this field must be a scalar field to be invariant. Since the scalar field has solved some of the fundamental problems of particle physics, such mechanism in quantum mechanics seems quite realistic.

Corresponding treatment of quantum phenomena on the basis of interaction of classical particles with a fluctuating classical scalar field was proposed in [12] as diffusion quantum mechanics (DQM).

In the present paper, after a brief description of DQM and a diffusion explanation of the main quantum phenomena, we will analyze in more detail its new consequences. Diffusion gravity, directly following from DQM as thermal diffusion in the inhomogeneous scalar field, will be considered in [13]. 
In Sections 2 and 3 the physical principles of DQM, the derivation of the formalism of quantum mechanics and quantum statistics are considered. In Section 4 some of new consequences of DQM are discussed.

\section{QUANTUM-MECHANICAL EVOLUTION AS CONSERVATIVE DIFFUSION}

\section{A. Physical principles of DQM}

The geometric postulate underlying classical and quantum mechanics, that physical space is continuous, homogeneous and isotropic, remains valid in DQM as well. The principle of relativity, as well as in classical and quantum mechanics, remains be the first physical principle of DQM.

If the behavior of an ensemble of quantum particles is similar to a specific diffusion of classical particles, then it is necessary to clear up in what this specificity consists of and how this type of diffusion can be described. Further, as in nonrelativistic quantum mechanics, we restrict ourselves by the nonrelativistic approximation in order to separate purely quantum effects from relativistic complications.

In the case of ordinary (dissipative) diffusion of a particle in a medium, gas or liquid, friction plays an essential role and the rest frame of this medium (or its part where the particles diffuse) is the preferred frame for this ensemble. Friction gradually decreases initial non-zero drift velocity and the ensemble becomes resting relative to the medium. In contrast to the dissipative diffusion, quantum phenomena occur similarly in all inertial frames, in strict accordance with the principle of relativity. Thus, the first specificity of the new kind of diffusion is that it must be frictionless, a conservative diffusion, which means the constancy of the drift velocity in an ensemble of free particles.

The principle of relativity and homogeneity of space and time lead to constancy and invariance of the diffusion coefficient $D$ determined by Fick's law, while the correspondence with classical mechanics requires its proportionality to the Planck constant $D=\hbar b$. At taking the proportionality constant as $b=1 / 2 m_{q}$, where $m_{q}$ is some "quantum-diffusion mass" of a particle, gives $D=\hbar / 2 m_{q}$. Experiments show that $m_{q}$ is equal to the inertial mass: $m_{q}=m_{i n}[14]$ and further we will write it as $m$.

In classical physics, there is an example of a medium whose fluctuations are invariant and occur similarly in all inertial frames. This is a fluctuating classical scalar field, where the time evolution of a localized ensemble of classical particles interacting with it is an example of conservative diffusion. In this case, if the initial probability density for an ensemble of free particles in the inertial frame $K$ had a Gaussian form with zero drift velocity $\mathbf{v}=0$, then in another inertial frame $K^{\prime}$, moving relative to $K$ with velocity $\mathbf{V}$, the center of this distribution will move with a constant drift velocity $\mathbf{v}^{\prime}=-\mathbf{V}$. Conversely, an ensemble in $K^{\prime}$ with the same distribution and $\mathbf{v}^{\prime}=0$ diffuses in $K^{\prime}$ as in $K$, but moves relative to $K$ with the drift velocity $\mathbf{v}=\mathbf{V}$.

Thus, to construct a diffusion theory of quantum phenomena, we need to consider the description of the motion of an ensemble of classical particles in a classical scalar field. Therefore, the postulate about the existence of a fluctuating classical scalar field, indirectly confirmed by experiments by the fact of quantum fluctuations of free microparticles, is the second physical principle of DQM. Then, as it will be shown below, a hydrodynamic picture, where corresponding diffusion is described by densities and fluxes only, without specifying its source and nature, allows one to derive the formalism of quantum mechanics and to explain quantum behaviour of diffusing classical particles.

\section{B. Kinematics and dynamics of DQM}

The classical particles interacting with a fluctuating classical scalar field move chaotically and the ensemble of one particle states is described by the probability density $\rho(\mathbf{x}, t)$ normalized to unity:

$$
\int \rho(\mathbf{x}, t) d^{3} x=1
$$

If $\rho(\mathbf{x}, t)$ is distributed inhomogeneously and its gradient is nonzero, then there arises a diffusion flux $\mathbf{j}_{D} \equiv \mathbf{u} \rho$, where $\mathbf{u}(\mathbf{x}, t)$ is the local diffusion velocity. As in any diffusion, $\mathbf{j}_{D} \sim-\nabla \rho$ and the flux obeys the diffusion law (Fick's law):

$$
\mathbf{j}_{D}=\mathbf{u} \rho=-D \nabla \rho=-\frac{\hbar}{2 m} \nabla \rho .
$$

This gives an expression for $\mathbf{u}$ and determines its properties:

$$
\mathbf{u}=-\frac{\hbar}{2 m} \frac{\nabla \rho}{\rho}, \quad \overline{\mathbf{u}}=\int \mathbf{u} \rho d^{3} x=0, \quad \int \mathbf{u}^{2} \rho d^{3} x \neq 0 .
$$

These properties of $\mathbf{u}$ lead to the "uncertainty relations" for the momentum of diffusion flux $\mathbf{p}_{\mathbf{u}}=m \mathbf{u}($ at $\overline{\mathbf{x}}=0)$ :

$$
\overline{\mathbf{p}_{\mathbf{u}}^{2}} \cdot \overline{\mathbf{x}^{2}} \geq\left|\overline{\mathbf{p}_{\mathbf{u}} \cdot \mathbf{x}}\right|^{2}=(m D)^{2}\left|\int \nabla \rho \cdot \mathbf{x} d^{3} x\right|^{2}=\frac{\hbar^{2}}{4} .
$$

These relations mean that the diffusion flux tends to reduce the degree of localization of the ensemble of particles around the centre of localization $\overline{\mathbf{x}}=0$. As smaller the localization area, as greater $\mathbf{u}$, directed opposite to $\nabla \rho$, and as greater the mean kinetic energy of the diffusion flux $\bar{U}_{u}$ :

$$
\bar{U}_{u}=\int \frac{\mathbf{p}_{\mathbf{u}}^{2}}{2 m} \rho d^{3} \mathbf{x}=\int \frac{\hbar^{2}}{8 m}\left(\frac{\nabla \rho}{\rho}\right)^{2} \rho d^{3} x .
$$

Thus, the flux energy $\bar{U}_{u} \sim \mathbf{p}_{\mathbf{u}}^{2} / 2 m$ is equal to the energy of localization $\bar{U}_{l o c} \sim(\nabla \rho / \rho)^{2}$ that was transferred to the ensemble during the localization most of the particles of the ensemble in a finite volume (by the walls of the box, by an external force, etc.): $\bar{U}_{u}=\bar{U}_{l o c}$. Since $\bar{U}_{l o c}$ depends on $\rho$ only, therefore, it is in fact the potential energy of localization. 
If the external force ceases to act, then this potential energy generates the diffusion flux which will delocalize the ensemble by leading to $\mathbf{u} \rightarrow 0, \rho \rightarrow \rho^{\prime} \sim$ const.

The local drift velocity $\mathbf{v}(\mathbf{x}, t)$ of the particle depends on two factors - the choice of an inertial frame and the velocity attained at acceleration in a potential $V$. At conservative diffusion, due to the absence of friction, $\mathbf{v}$ is similar to the velocity in classical mechanics. Therefore, there is an action function of the drift $S$ related by the drift momentum $\mathbf{p}_{\mathbf{v}}=m \mathbf{v}$ as:

$$
\mathbf{p}_{\mathbf{v}}=m \mathbf{v}=\nabla S, \quad \mathbf{v}=\nabla S / m .
$$

Since the total local velocity in the ensemble, averaged under local fluctuations, is $\mathbf{v}+\mathbf{u}$, the Hamilton function $H$, including the interaction potential $V$, has the form:

$$
\begin{aligned}
H & =\int\left(\frac{m c^{2}}{\sqrt{1-(\mathbf{v}+\mathbf{u})^{2} / c^{2}}}+V\right) \rho d^{3} x \simeq \\
& \simeq \int\left(m c^{2}+\frac{\mathbf{p}_{\mathbf{v}}^{2}}{2 m}+\frac{\mathbf{p}_{\mathbf{u}}^{2}}{2 m}+V\right) \rho d^{3} x .
\end{aligned}
$$

Then, by using (3) and (6), we obtain:

$$
H \simeq \int\left(m c^{2}+\frac{1}{2 m}(\nabla S)^{2}+\frac{\hbar^{2}}{8 m}\left(\frac{\nabla \rho}{\rho}\right)^{2}+V\right) \rho d^{3} x .
$$

Here the presence of the fluctuating classical scalar field is manifested in the appearance of the probability density $\rho$, the energy of diffusion flux $U_{u}$ and the rest energy $m c^{2}$, which below will be interpreted as the "thermal energy" of particle's fluctuations.

\section{Canonical formalism for DQM}

To describe the motion of a classical particle in empty space, its coordinates $\mathbf{x}(t)$ and momenta $\mathbf{p}=m \dot{\mathbf{x}}$ are required. But in the case of conservative diffusion in the fluctuating field, it is more convenient the hydrodynamic description with the Hamilton function $H$ (8), where the role of a generalized coordinate plays $\rho$ as some field variable.

Since $H$ in (8) contains, besides $\rho$, only $S$ associated with the drift velocity $\mathbf{v}(6)$, one can take $S$ as a generalized momentum, canonically conjugate to $\rho$. In the phase space $(\rho, S)$ the Poisson brackets are defined as [7]:

$$
\begin{gathered}
\{A, B\}=\int\left(\frac{\delta A}{\delta \rho} \frac{\delta B}{\delta S}-\frac{\delta B}{\delta \rho} \frac{\delta A}{\delta S}\right) d^{3} x, \\
\left\{\rho(x, t), S\left(x^{\prime}, t\right)\right\}=\delta\left(x-x^{\prime}\right), \\
\left\{\rho(x, t), \rho\left(x^{\prime}, t\right)\right\}=\left\{S(x, t), S\left(x^{\prime}, t\right)\right\}=0 .
\end{gathered}
$$

The corresponding canonical equations take the form:

$$
\frac{\partial S}{\partial t}=\{S, H\}=-\frac{\delta H}{\delta \rho}, \quad \frac{\partial \rho}{\partial t}=\{\rho, H\}=\frac{\delta H}{\delta S} .
$$

At substituting $H$ from (8) into (11) and transformation by inserting full derivative (insensitive due to integration):

$$
\left(\frac{\nabla \rho}{\rho}\right)^{2}+2 \nabla\left(\frac{\nabla \rho}{\rho}\right)=4 \frac{\Delta\left(\rho^{1 / 2}\right)}{\rho^{1 / 2}},
$$

the canonical equations (11) take the form:

$$
\begin{gathered}
\frac{\partial S}{\partial t}+\left(\frac{(\nabla S)^{2}}{2 m}+V\right)-\frac{\hbar^{2}}{2 m} \frac{\Delta\left(\rho^{1 / 2}\right)}{\rho^{1 / 2}}=0, \\
\frac{\partial \rho}{\partial t}+\nabla(\rho \cdot \nabla S / m)=0 .
\end{gathered}
$$

Here (13) is the well-known Hamilton-Jacobi-Madelung equation [1], while (14) is the continuity equation.

The canonical equations (13)-(14) are nonlinear under the canonical pair $\rho, S$. But they can be linearized using the canonical transformation:

$$
\psi=\rho^{1 / 2} e^{i S / \hbar},
$$

performing a transition from the phase space $(\rho, S)$ to the phase space $\left(\psi^{*}, \psi\right)$ formed by the complex conjugate probability amplitudes $\psi$ and $\psi^{*}$. As the result, two realvalued canonical equations (13)-(14) form one a complex equation, which is the Schrödinger equation [1]:

$$
i \hbar \frac{\partial \psi}{\partial t}=-\frac{\hbar^{2}}{2 m} \Delta \psi+V \psi
$$

Since one of the canonical equations (14) is the continuity equation, where $\partial_{t} \rho$ is related by $S$, this confirms the choice of $S$ as a canonical momentum, since it represents $\partial_{t} \rho$ in $H$ through one of the canonical equations.

\section{Diffusion algebra of observables and operator algebra}

In the hydrodynamic description of conservative diffusion, an observable $A$ is a function of $\rho$ and $S$ in the phase space $(\rho, S)$ and in the phase space $\left(\psi^{*}, \psi\right)$ it leads to the bilinear form for the corresponding function $A\left(x, x^{\prime}\right)=A\left(x^{\prime}, x\right)$ [7]:

$$
A(\rho, S)=\iint \psi^{*}(x) A\left(x, x^{\prime}\right) \psi\left(x^{\prime}\right) d^{3} x d^{3} x^{\prime} .
$$

The observables are real-valued and therefore it is possible to introduce a Hermitian operator $\hat{A}$ acting on the amplitude as:

$$
(\hat{A} \psi)(x)=\int A\left(x, x^{\prime}\right) \psi\left(x^{\prime}\right) d^{3} x^{\prime} .
$$

Then the bilinear form (17) takes the standard form:

$$
A(\rho, S)=\int \psi^{*}(x) \hat{A} \psi(x) d^{3} x \equiv\langle\psi, \hat{A} \psi\rangle .
$$

In particular, the Poisson bracket of two observables (9) takes the form of a bilinear form from corresponding commutator:

$$
\{A, B\}=(1 / i \hbar)\langle\psi,[\hat{A}, \hat{B}] \psi\rangle
$$

Particularly, the mean value of the particle's coordinate along the trajectory, as an observable, can be written as: 


$$
\overline{\mathbf{x}}(t)=\int \rho \mathbf{x} d^{3} x=\langle\psi, \mathbf{x} \psi\rangle
$$

The translational invariance $\mathbf{x}^{\prime}=\mathbf{x}+\mathbf{a}$ leads to the conservation of momentum, for which there is a standard operator expression:

$$
\overline{\mathbf{p}}=\langle\psi, \hat{\mathbf{p}} \psi\rangle, \quad \hat{\mathbf{p}}=(\hbar / i) \nabla
$$

In the diffusion picture, this observable has the form:

$$
\overline{\mathbf{p}}(\rho, S)=\int\left(\mathbf{p}_{\mathbf{v}}+i \mathbf{p}_{\mathbf{u}}\right) \rho d^{3} x=\int \rho \nabla S d^{3} x
$$

and acts as the generator of translations for the canonical pair:

$$
\begin{gathered}
\delta \rho=\delta \mathbf{a} \cdot\{\rho, \overline{\mathbf{p}}\}=\delta \mathbf{a} \cdot \frac{\delta \overline{\mathbf{p}}}{\delta S}=-\delta \mathbf{a} \cdot \nabla \rho, \\
\delta S=\delta \mathbf{a} \cdot\{S, \overline{\mathbf{p}}\}=-\delta \mathbf{a} \cdot \frac{\delta \overline{\mathbf{p}}}{\delta \rho}=-\delta \mathbf{a} \cdot \nabla S .
\end{gathered}
$$

The time derivative of an observable in two pictures is:

$$
\frac{d A}{d t}=\frac{\partial A}{\partial t}+\{A, H\}=\frac{\partial A}{\partial t}+\frac{1}{i \hbar}\langle\psi,[\hat{A}, \hat{H}] \psi\rangle .
$$

\section{QUANTUM STATISTICS AS CONSEQUENCE OF DQM}

\section{A. Classical statistics and two forms of quantum statistics}

In statistical mechanics, studying systems of a very large number of particles, the source of fluctuations is chaotic collisions (or other forms of chaotic interactions) in the manyparticle system. The phase space of the system of $N$ particles is divided into small cells, and to determine $P\left\{n_{i}\right\}$, the probabilities of filling of $M$ states with filling numbers $\left\{n_{i}\right\}, i=1, \ldots, M$, one needs in the number of ways (statistical weight) $\Delta \Gamma$ with which these states can be filled [15].

In classical statistical mechanics, firstly, particles in empty space are considered distinguishable, and secondly, the probabilities $w_{i}$ of all $M$ states are assumed the same and equal to $w=1 / M$. The large number of states, $N \ll M$, and the small average occupation numbers of states allow one to suppose that particles are distributed independently. If each of $N$ particles are placed in one of the $M$ states, this gives $M^{N} n_{1} ! n_{2} ! \ldots n_{M}$ ! possible distributions. It also should be divided to $N$ !, the number of permutations, and then the inverse of the statistical weight $\Delta \Gamma_{M B}$ of the distribution of $N$ particles to $M$ states gives $P_{M B}\left\{n_{i}\right\}$ :

$$
P_{M B}\left\{n_{i}\right\}=\frac{1}{\Delta \Gamma_{M B}}=\frac{M^{-N} N !}{n_{1} ! n_{2} ! \ldots n_{M} !} .
$$

The requirement that the entropy $S=\ln \Delta \Gamma$ at equilibrium is maximal leads to the classical distribution for an equilibrium gas, the Maxwell-Boltzmann (MB) distribution $\bar{n}_{i}=e^{-E_{i} / k T}$.

In the formalism of quantum mechanics, the probabilities of realizing different states of a system of particles were also considered the same and the difference from classical statistics was reduced only to two properties: a) the particles were considered indistinguishable, and b) the occupation numbers should not be small. It turned out that the property of indistinguishability changes the statistical weight of states in such a way that, instead of classical statistical mechanics, there appears one of two forms of quantum statistics. For the probability amplitudes symmetric under the permutations of pairs of particles this leads to the Bose-Einstein (BE) statistics with $\bar{n}_{i}=\left(e^{E_{i} / k T}-1\right)^{-1}$ and for antisymmetric ones - to the Fermi-Dirac (FD) statistics with $\bar{n}_{i}=\left(e^{E_{i} / k T}+1\right)^{-1}[15]$.

\section{B. Quantum statistics from the conservative diffusion of classical particles}

In DQM, the particles are classical and therefore remain distinguishable, but nevertheless, both forms of quantum statistics arise. Here in a gas of microparticles there are two types of probabilistic laws - the mutual collisions of particles lead to the statistical laws similar to classical statistics, and the fluctuations of particles in the scalar field between collisions lead to the quantum properties of the system. Therefore, if for classical particles in empty space all states were equally probable, then for the same particles fluctuating between collisions the probabilities should not be the same.

This difference is easier to see for antisymmetric states, when in one state there can be no more than one particle. This property is not related to the many-particle nature of the system and takes place even for a system of two particles, i.e. this is precisely the consequence of fluctuations in the scalar field. Therefore, for such systems, instead of the number of distributions $M^{N}$, it is necessary to take only the number of combinations of $M$ elements with respect to $N$ and, instead of (27), combinatorics gives

$$
P_{F D}\left\{n_{i}\right\}=\frac{1}{\Delta \Gamma_{F D}}=\frac{N !(M-N) !}{M !}, \quad \bar{n}_{i}=\frac{1}{e^{E_{i} / k T}+1},
$$

i.e. MB distribution becomes replaced by FD distribution.

The reason why BE statistics will appear in the system of classical particles was discovered by Tersoff and Bayer [16]. Instead of the equal probability condition, they took a weaker condition and obtained BE distribution in classical physics. These probabilities were assumed as having arbitrary values $w_{1}, \ldots, w_{M}$, limited only by the usual properties of probabilities $0 \leq w_{i} \leq 1, \sum_{i=1}^{M} w_{i}=1$. Therefore, one needs to average over all their permissible values, i.e. integrate over $w_{i}$, but with the restriction that the sum of the probabilities must be equal one.

In [16], the question of why equiprobability should be replaced by averaging over arbitrary probabilities was remained open, since there is no reason for this in a classical ideal gas, while in standard quantum mechanics particles are not classical. But DQM explains this naturally, since there are additional fluctuations between collisions. This gives a random spread and one must, firstly, to take into account it and, secondly, to average over all these deviations.

Thus, in DQM with distinguishable particles, the equal probability condition should be replaced by averaging with 
weight coefficients, i.e. each state has a weighted probability $\left\{w_{i}\right\}$ with the usual constraints on the probabilities. As a result, the probability in (27) becomes replaced by:

$$
P\left\{n_{i}\right\}=N !\left[\prod_{i=1}^{M} \frac{1}{n_{i} !} \int_{0}^{1} d w_{i} \cdot w_{i}^{n_{i}}\right] \delta\left(1-\sum_{i=1}^{M} w_{i}\right) .
$$

Here the integrals should be calculated by including into their limits the constraints on $w_{i}$, i.e. by taking $0 \leq w_{2} \leq 1-w_{1}$ at $M=2$ etc. Each following integral has the form

$$
\int_{0}^{a} d w \cdot w^{m}(a-w)^{n}
$$

and, calculated recursively, finally gives the BE result:

$$
P_{B E}\left\{n_{i}\right\}=\frac{1}{\Delta \Gamma_{B E}}=\frac{N !(M-1) !}{(M+N-1) !}, \quad \bar{n}_{i}=\frac{1}{e^{E_{i} / k T}-1} .
$$

Thus, in the gas of distinguishable particles, additionally fluctuating between collisions, it takes place BE distribution. Correlations between particles in different states, absent in classical mechanics, appear due to interaction of particles with globally defined scalar field. Indistinguishability of particles in quantum statistics appears effectively as a consequence of accounting these additional fluctuations and the correlations between particles through the states of scalar field.

The Pauli exclusion principle for systems of particles described by an antisymmetric wave function is a new property of quantum fluctuations. Independently of details of the relationship between spin and statistics, DQM describes processes by the probability amplitudes and thus it allows such states, regardless of the reasons for their occurrence.

\section{SOME NEW CONSEQUENCES OF DQM}

\section{A. Fluctuations of particles with light velocity and fluctuation energy as rest energy}

Microparticles, moving in the fluctuating classical scalar field, have a fluctuation velocity $\mathbf{u}_{q}$ with vanishing mean value $\overline{\mathbf{u}}_{q}=0$ and a nonzero rms $\overline{\mathbf{u}_{q}^{2}}>0$. The latter should be invariant, and therefore, constant: $\overline{\mathbf{u}_{q}^{2}}=$ const. In condensed media it would be proportional to temperature: $\overline{\mathbf{u}_{T}^{2}} \sim T$.

Since the fluctuations of classical particles in the scalar field occur similarly in any inertial frame, their velocity between interactions with the field must be invariant and, therefore, equal to the light velocity $\left|\mathbf{u}_{q}\right|=c$. Therefore, the particles are initially massless, and only their random walk in space due to interaction with the field leads to the rest energy.

An ensemble of free particles in DQM, as well as in quantum mechanics, is described by the plane wave: $\psi \sim \exp [i(\mathbf{p x}-E t) / 2 m D]$, i.e. here $S(\mathbf{x}, t)=\mathbf{p x}-E t$ and $\rho^{1 / 2}=$ const. Since $\mathbf{u} \sim \nabla \rho=0$, then $\mathbf{p}_{u}=0, \bar{U}_{u}=0$. Thus, for free particles, there is no localization energy and, at taking $\mathbf{v}=0$ (in the rest frame), the total energy becomes reduced to the "thermal" energy only: $U_{m} \sim \mathbf{u}_{q}^{2} \sim c^{2}$.

This part of the energy of particles is well known as their rest energy $E_{0}=m c^{2}$ in (7)-(8), appeared in the relativistic theory. Therefore, we can identify it with the constant thermal energy of particle's fluctuations in the fluctuating field $U_{m}$ :

$$
U_{m}=m c^{2}, \quad m=U_{m} / c^{2} .
$$

The mass of the particle $m$ in DQM, therefore, appears as the fluctuation energy $U_{m}$ divided to $c^{2}$ and describes intensity of its interactions with the fluctuating background field. This mass then appears in the diffusion coefficient: $D=\hbar / 2 m$.

From this point of view, photon is massless as moving rectilinearly. The fact that its angular momentum is quantized means that, its angular momentum nevertheless fluctuates.

Thus, if relativistic mechanics introduced into physics the invariance of the light velocity and then the rest energy, playing a key role in particle physics, then DQM allows for a deeper understanding of their physical meaning.

\section{B. New mass generation mechanism}

In the Standard Model of particle physics (SM), the rest energy of a particle appears as potential energy of its interaction with the vacuum condensate of the scalar field. Different coupling constants lead to different masses.

In DQM, the rest energy of a particle manifests itself as the kinetic energy of its fluctuations, occurring due to its interaction with the scalar field. Since different interaction constants lead to different intensity of these fluctuations, and hence to different rest energies (masses), this treatment is in agreement with the SM treatment of mass generation.

At averaging at a large, but finite time interval $T$ the mean value of the fluctuating part of the classical scalar field $\phi(\mathbf{r}, t)$ vanishes in any point $\mathbf{r}$, while its rms remains non-zero:

$$
\frac{1}{T} \int_{0}^{T} \phi(\mathbf{r}, t) d t=0, \quad \frac{1}{T} \int_{0}^{T}|\phi(\mathbf{r}, t)|^{2} d t=\eta^{2}(\mathbf{r}), \quad \eta>0 .
$$

The Lagrangian of the field contains a covariant derivative $\left|D_{\mu} \phi\right|^{2}=\left|\left(\partial_{\mu}-i g^{(a)} A_{\mu}^{(a)} / 2\right) \phi\right|^{2}$, including gauge fields $A_{\mu}^{(a)}$. Therefore, Eq. (33) leads to the mass $m^{(a)}=g^{(a)} \eta / 2$ for $A_{\mu}^{(a)}$ and this result for gauge fields is exactly the same as in SM.

However, for fermions the interaction term with the scalar field may include linear and quadratic under $\phi$ terms and only the latter, through rms, leads to the mass of the particles.

Notice, that in DQM the fluctuating part of $\phi$ leads to the quantum fluctuations, while its rms generates particle's mass. Thus, DQM contains a natural mass generation mechanism, the results of which are in full accordance with the SM results for gauge bosons. It is important, that this new mechanism requires the fluctuations of the scalar field only, while spontaneous symmetry breaking is not necessary. 


\section{Diffusion mechanism for small mass of composite particles}

SM contains a large number of primary particles, formally taken as pointlike. However, their possible substructure at small distances is not only allowed by SM, but it is even desirable to exclude internal contradictions. Firstly, loop contributions of the scalar field grow as degrees of cutoff energy, being very large long before the Planck scale, when gravity is negligible and cannot be a natural regulator. This problem will be solved only if the scalar field is effective and the scalar particle is composite. Secondly, even if the first generation of fermions is primary, it is difficult to accept the primary character of the next two generations.

In this connection, composite models of SM particles were proposed (see, for example, [17]). However, these models have a serious difficulty - the mass problem. Smallness of the localization region leads to high kinetic energies of subparticles due to the uncertainty relations, while the observed masses of SM particles are sufficiently smaller. Even if the binding energy of subparticles mainly compensates their kinetic energy, the mass difference between the excited states remains be very large and thus the next generations of fermions cannot be the excited states. Attempts to solve the mass problem in quantum mechanics were unsuccessful. Because of this problem and some other technical difficulties, composite models seemed hopeless and practically never developed during last decades.

However, DQM, as a theory going beyond the formalism of quantum mechanics, may reanimate the composite models of SM particles, since it has a mechanism for the formation of composite particles of small mass, similar to the mechanism of chemical reactions between impurities [13]. This mechanism avoids the constraints of the uncertainty relation and thereby can solve the mass problem.

If two types of impurity atoms in a gas began to diffuse so that the mean distance between the impurity atoms larger than the mean free path of medium's atoms, then the diffusion strongly suppresses the chemical reactions between these impurities. However, in any case when two impurity atoms happen to be at a distance less than the mean free path, they succeed to form a molecule during the free path time. Then this molecule (composite particle) diffuses as all other atoms, but by the increased mass.

In [18], it was proposed to use the same mechanism of the formation of composite particles of small mass in DQM and then this mechanism was applied to the simplest of subparticle

[1] Madelung, E. (1926) Zs. Phys., 40, 322.

[2] Ehrenfest, P. (1927) Zs. Phys., 45, 455.

[3] Schrödinger E. (1930) Sitz. Preus. Akad. Wiss., 418; (1932) Ann. I.H.P. 2(4) 269.

[4] Fürth, R. (1933) Zs. Phys., 81, 143.

[5] Fényes, I. (1952). Zs. Phys., 132, 81.

[6] Nelson, E. (1966) Phys. Rev., 150, 1057; (1985) Quantum Fluctuations. Prins. U. P.

[7] Guerra, F., Marra R. (1983) Phys. Rev. D 28, 1916.

[8] Weizel, W. (1953) Zs. Phys., 135, 270.

[9] Grabert, H. et al (1979) Phys. Rev. A19, 2440. models - the rishon model [17]. This mechanism, if it appears realistic, would practically solve the mass problem, making the composite models of SM particles consistent. This can also simplify further unification of fundamental fields.

\section{Gravitation as a "thermal" diffusion in the inhomogeneous background field}

The main new property of DQM, discriminating it from quantum mechanics, is the energy-momentum exchange between the scalar field and diffusing particles. The most important consequence of this fact is the appearance of thermal diffusion due to the inevitable formation of inhomogeneities in the background scalar field. This happens because a part of the field's energy becomes transferred to any particle as the energy of its fluctuations (rest energy) $m c^{2}$, and, as the result, the energy of the scalar field around the particle should decrease to the same value $m c^{2}$.

Thus, a value of rms $\eta^{2}(r)$ from (33) becomes less than at spatial infinity $r \rightarrow \infty$ and, by defining $\eta(\infty) \equiv \eta_{0}$, we have:

$$
\eta\left(r_{1}\right)<\eta\left(r_{2}\right)<\eta_{0}, \quad r_{1}<r_{2}<\infty .
$$

This basic property of DQM turned out to be the desired physical mechanism of gravity and this consequence of DQM is considered in more details in the next paper [13].

\section{CONCLUSIONS}

Thus, the postulate about the existence of a fluctuating classical scalar field as the source of quantum fluctuations of classical particles, leads to DQM. DQM then reproduces the formalism of quantum mechanics and leads to several new consequences, including gravitation. The linear structure of quantum mechanics and its unusual consequences, such as particle-wave duality, entanglement etc., follow from the specifics of conservative diffusion.

The main tasks for further research in the framework of DQM are the causes and mechanisms of fluctuations of the scalar field, while the postulating of these fluctuations as the observational fact leads to a simple and clear physical picture of both quantum and gravitational phenomena, sufficiently simplifying the situation in the foundations of physics.

[10] Feynman, R. P., Hibbs, A. R. (1965) Quantum Mech. and Path Int. McG.-H.

[11] Bohr, N. (1949) “A. Einstein, philosopher-scientist”, 201.

[12] Zakir, Z. (2020) Quant. and Grav. Phys., 1:003-7129.

[13] Zakir, Z. (2021) Quant. and Grav. Phys., 2:014-7610.

[14] Smolin, L. (1986) Phys. Lett., 113A, 408.

[15] Landau, L. D., Lifshitz, E. M. (1980) Stat. Physics. 1. Per. P.

[16] Tersoff, J., Bayer, D. (1983) Phys. Rev. Lett. 50, 553.

[17] Harari, H. (1979) Phys. Lett. 86B, 83; Shupe, M.A. (1979) Phys. Lett., 86B, 87.

[18] Zakir, Z. (2016) Theor. Phys., Astroph. and Cosmol. 11, 1. 